\title{
Natura@economía
}

ISSN 2226-9479 (Versión electrónica) Website: http://revistas.lamolina.edu.pe/index.php/neu

\section{Valoración económica ambiental de los bofedales del distrito de Pilpichaca, Huancavelica, Perú}

\section{Economic environmental assessment of the bofedales of the district of Pilpichaca, Huancavelica, Peru}

\author{
Marianella Crispin Cunya ${ }^{1}$; Luis Alberto Jimenez Diaz ${ }^{1 *}$
}

${ }^{1}$ Universidad Nacional Agraria La Molina, Lima, Perú.

Recepción: 31 enero del 2018Aceptación: 15/04/2019

\begin{abstract}
Resumen
El objetivo de la presente investigación fue Valorar los Servicios Ambientales de provisión de agua y almacenamiento de agua y carbono en el distrito de Pilpichaca, provincia de Huaytará departamento de Huancavelica; para determinar la importancia económica y ambiental, se consideró las 8 369,67 ha de bofedales definidos en el Zonificación Económica Ecológica del Gobierno Regional de Huancavelica, publicado en el año 2014; también, se consideró el número total de llamas y alpacas por comunidad involucrada de acuerdo al Gobierno Regional de Huancavelica para el año 2013. Para la valorización económica de los bofedales se utilizó el concepto de Valor Económico Total. Como resultado del análisis, el valor del Servicio Ambiental Provisión de agua es mayor en comparación con los Servicios ambientales de Almacenamiento de agua y carbono en el suelo de los bofedales.
\end{abstract}

Palabras clave: humedal; Perú; Medio Ambiente; Valoración económica.

\begin{abstract}
The objective of the present investigation was to Assess the Environmental Services of water supply and water and carbon storage in the district of Pilpichaca, province of Huaytará department of Huancavelica; to determine the economic and environmental importance. It was considered 8369,67 ha of bofedales defined in the Economic Ecological Zoning of the Regional Government of Huancavelica, published in 2014; Also, the total number of llamas and alpacas per community involved was considered according to the Regional Government of Huancavelica for the year 2013. For the economic valuation of the bofedales, the concept of Total Economic Value was used. As a result of the analysis, the value of the Environmental Service Water provision is greater in comparison with the Environmental Services of Storage of water and carbon in the soil of the bofedales.
\end{abstract}

Keywords: wetland; Peru; Environment; Economic valuation.

Forma de citar el artículo: Crispin, M.; Jimenez, L. 2019. Valoración económica ambiental de los bofedales del distrito de Pilpichaca, Huancavelica, Perú. Natura@economía 4(1): 1-13 (2019).

(C) Facultad de Economía y Planificación, Universidad Nacional Agraria La Molina, Lima, Perú. 


\section{Introducción}

Los bofedales, son una clase de humedal en zonas altas donde existen praderas pantanosas y frías alrededor de cuerpos de agua como lagos glaciares, manantiales, ríos riachuelos; donde el drenaje es pobre, el suelo es mineralizado y arcilloso, el cual permite almacenar agua durante todo el año, generando un hábitat para pastos (Gil, 2011), que son característicos de la puna alta y por consiguiente de gran importancia para el pastoreo de altura (Custred, 1997). En esta zona la precipitación es mayor y la evaporación menor; este tipo de humedal juega un papel importante en la regulación del ciclo hídrico y en el almacenamiento de carbono.

Las características generales de los bofedales son: almacenar agua, es un sistema frágil, pueden ser fácilmente alterados, tienen una morfología almohadillada, poseen aguas mineralizadas, tienen fluctuaciones climáticas que van desde los
-14 a $20^{\circ} \mathrm{C}$; presentan inundación de carácter permanente, están ligados a emanaciones naturales de agua, se originan en las cabezas de casi todos los ríos de la zona, el 70 a $75 \%$ del total anual de precipitaciones se producen durante los meses de diciembre a marzo; el promedio del total de horas de sol es de $2971 \mathrm{~h}$ y durante la noche en el invierno la temperatura desciende a varios grados bajo $0^{\circ}$, de modo que el agua de los bofedales se congela (Gil, 2011).

Asimismo, el Programa de las Naciones Unidas para el Medio Ambiente (PNUMA) considera ocho servicios ambientales de los bofedales (Tabla 1).

\section{Experiencia sobre valoración económica de agua y carbono en los bofedales de los páramos ecuatorianos}

Castro (2011) indica que los bofedales y lagunas tienen una alta importancia en el ciclo hidrológico del páramo a través de la provisión y regulación hídrica. Al igual que

Tabla 1: Servicios ambientales y funciones ecosistémicos de los bofedales

\begin{tabular}{|c|c|c|}
\hline Servicios ambientales & Funciones & Ejemplos \\
\hline Regulación de Gases & $\begin{array}{l}\text { Regular la composición química } \\
\text { atmosférica. }\end{array}$ & Balance $\mathrm{CO}_{2} / \mathrm{O}_{2}$. \\
\hline Regulación de Clima & $\begin{array}{l}\text { Regular la temperatura global, } \\
\text { precipitación y otros procesos } \\
\text { climáticos locales y globales. }\end{array}$ & $\begin{array}{l}\text { Regulación de gases de efecto } \\
\text { invernaderos (Captura de Carbono). }\end{array}$ \\
\hline $\begin{array}{l}\text { Regulación } \\
\text { disturbios }\end{array}$ & $\begin{array}{l}\text { Capacidad del ecosistema de dar } \\
\text { respuesta y adaptarse a fluctuaciones } \\
\text { ambientales. }\end{array}$ & $\begin{array}{l}\text { Protección de sequías, respuesta del } \\
\text { hábitat a cambios ambientales. }\end{array}$ \\
\hline Oferta de agua & Almacenar y retener agua. & $\begin{array}{l}\text { Provisión de agua proveniente de } \\
\text { acuíferos. }\end{array}$ \\
\hline $\begin{array}{lr}\text { Retención } & \text { de } \\
\text { sedimentos } & \text { y control } \\
\text { de erosión } & \end{array}$ & $\begin{array}{l}\text { Retención del suelo dentro del } \\
\text { ecosistema. }\end{array}$ & $\begin{array}{l}\text { La cobertura de bofedal previene la } \\
\text { pérdida de suelo por viento. } \\
\text { Almacenar de agua en los bofedales. }\end{array}$ \\
\hline Refugio de especies & $\begin{array}{l}\text { Hábitat para poblaciones residentes y } \\
\text { migratorias. }\end{array}$ & $\begin{array}{l}\text { Semilleros, hábitat de especies } \\
\text { migratorias, locales. }\end{array}$ \\
\hline Materia prima & $\begin{array}{l}\text { Producción bruta primaria extractable } \\
\text { de materias primas. }\end{array}$ & Producción de forrajes. \\
\hline Recreación & $\begin{array}{l}\text { Proveer oportunidades para actividades } \\
\text { recreacionales. }\end{array}$ & Ecoturismo. \\
\hline Cultural & $\begin{array}{l}\text { Proveer oportunidades para usos no } \\
\text { comerciales. }\end{array}$ & $\begin{array}{l}\text { Estética, artística, educacional, } \\
\text { espiritual, valores científicos del } \\
\text { ecosistema. }\end{array}$ \\
\hline
\end{tabular}


un cambio del uso del suelo tendrá un alto impacto tanto en la calidad de agua como en la regulación. Por ello, la valoración económica busca medir los beneficios de los servicios ambientales brindados por los bofedales, con la finalidad de promover la adopción de decisiones más equilibradas que faciliten y mejoren el uso racional y el manejo/gestión de los bofedales; para ello recurre a la valoración de los servicios ambientales.

En dicho estudio, se analizó dos escenarios (Tabla 2), una es la zona natural de Oña-Nabón-Saraguro-Yacuambi, que presenta un valor económico del servicio ambiental de provisión de agua para 218 ha de bofedal de US\$ 21 725,12/año, que da un Valor Actual Neto a perpetuidad de US\$ 344,5 miles. El valor del servicio de almacenamiento de agua en los bofedales es de US\$ 696,9 miles; que representa un valor de 3 196,8 \$/ha. El valor del servicio ambiental de almacenamiento de carbono en el suelo de los bofedales es de 13 340,62 \$/ha; que representa un valor total de US\$ 2,9 millones para toda la superficie de bofedales; de la integración de los tres Servicios Ambientales se obtiene un valor total de US\$ 3,9 millones. Por otro lado, en la zona intervenida Frente Suroccidental de Tungurahua, el valor económico del servicio ambiental de provisión de agua de las 1971 ha de bofedales de la zona de estudio es de US\$ 196,4 miles/año, que en Valor Actual Neto a perpetuidad da un valor de US\$ 2,9 millones. El valor del servicio de almacenamiento de agua en los bofedales es de US\$ 6,5 millones; que representa un valor de 3 299,21 \$/ha. El valor del servicio ambiental de almacenamiento de carbono en el suelo de los bofedales es de 7787,26 $\$ /$ ha; lo cual representa un valor total de

Tabla 2: Valoración económica de los Servicios ambientales de los bofedales

\begin{tabular}{lcc}
\hline $\begin{array}{c}\text { Servicios } \\
\text { ambientales }\end{array}$ & Natural (Oña) & $\begin{array}{c}\text { Natural } \\
\text { Tungurahua }\end{array}$ \\
\hline $\begin{array}{l}\text { Provisión de agua } \\
\text { Almacenamiento } \\
\text { de agua }\end{array}$ & $\$ 6949535,26$ & $\$ 2918618,29$ \\
$\begin{array}{l}\text { Almacenamiento } \\
\text { de carbono }\end{array}$ & $\$ 29082562751,36$ \\
\hline Valor total & $\$ 3949712,38$ & $\$ 24770053,66$ \\
\hline
\end{tabular}

Fuente: Castro (2011)
US\$ 15,3 millones para toda la superficie de bofedales. De la integración de los tres Servicios Ambientales en la superficie de 1971 ha de bofedales se generan un valor total de US\$24,8 millones.

En la Figura 1, se muestra el análisis proporcional, donde se puede apreciar que el valor del servicio de almacenamiento de carbono es el más importante en comparación con los servicios ambientales de almacenamiento y provisión de agua.

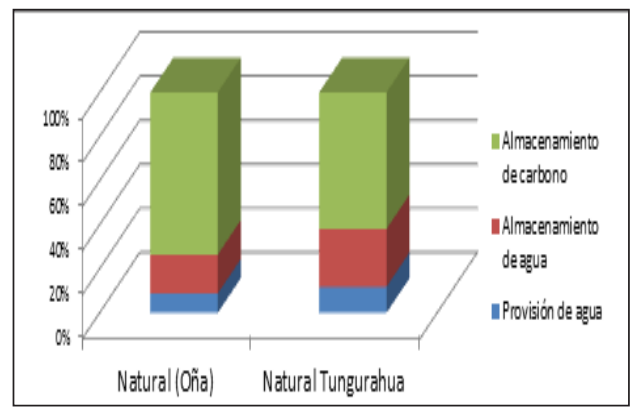

Figura 1: Integración de la valoración económica de los servicios ambientales

Fuente: Castro (2011)

\section{Los humedales y la convención RAMSAR}

La Secretaria de la Convención de Ramsar (2013) define como humedales "extensiones de marismas, pantanos y turberas o superficies cubiertas de aguas, sean estas de régimen natural o artificial, permanentes o temporales, estancadas o corrientes, dulces, salobres o saladas, incluidas las extensiones de agua marina cuya profundidad en marea baja no exceda de seis metros", asimismo, "zonas ribereñas o costeras adyacentes, así como las islas o extensiones de agua marina de una profundidad superior a los seis metros en marea baja, cuando se encuentren dentro del humedal". Existen cinco tipos morfológicos de sistemas de humedales los cuales son: Marinos (humedales costeros, inclusive lagunas costeras, costas rocosas y arrecifes de coral); Estuarinos (incluidos deltas, marismas de marea y manglares); Lacustres (humedales asociados con lagos); Ribereños (humedales adyacentes a ríos y arroyos); y Palustres (es decir, "pantanosos" - marismas, pantanos y ciénagas). En el Perú se lleva declarado un total de 13 sitios RAMSAR (Tabla 3), los cuales en su mayoría incluye reservas nacionales. 
Tabla 3: Listado de sitios Ramsar del Perú

\begin{tabular}{|c|c|c|c|c|c|}
\hline $\mathrm{N}^{\circ}$ Ramsar & Sitio Ramsar & Departamento & Área (ha) & $\begin{array}{l}\text { Fecha de } \\
\text { adhesión }\end{array}$ & Coordenadas \\
\hline 545 & PER-01 Reserva Nacional de Paracas & Ica & 335000 & $30 / 03 / 1992$ & $13^{\circ} 55^{\prime} \mathrm{S} 76^{\circ} 15^{\prime} \mathrm{O}$ \\
\hline 546 & PER-02 $\begin{array}{l}\text { Reserva Nacional Pacaya } \\
\text { Samiria }\end{array}$ & Loreto & 2080000 & $30 / 03 / 1992$ & $05^{\circ} 15^{\prime} \mathrm{S} 74^{\circ} 40^{\prime} \mathrm{O}$ \\
\hline 547 & $\begin{array}{l}\text { PER-03 Santuario Nacional Lagunas } \\
\text { de Mejía }\end{array}$ & Arequipa & 691 & $30 / 03 / 1992$ & $17^{\circ} 08^{\prime} \mathrm{S} 71^{\circ} 51^{\prime} \mathrm{O}$ \\
\hline 881 & PER-04 Lago Titicaca (área peruana) & Puno & 460000 & $20 / 01 / 1997$ & $15^{\circ} 50^{\prime} \mathrm{S} 69^{\circ} 30^{\prime} \mathrm{O}$ \\
\hline 882 & PER-05 Reserva nacional de Junín & $\begin{array}{l}\text { Junín } \\
\text { Pasco }\end{array}$ & 53000 & $20 / 01 / 1997$ & $13^{\circ} 55^{\prime} \mathrm{S} 76^{\circ} 15^{\prime} \mathrm{O}$ \\
\hline 883 & $\begin{array}{ll}\text { PER-06 } & \begin{array}{l}\text { Santuario Nacional Los } \\
\text { Manglares de Tumbes }\end{array}\end{array}$ & Tumbes & 2972 & $20 / 01 / 1997$ & $03^{\circ} 25^{\prime} \mathrm{S} 80^{\circ} 17^{\prime} \mathrm{O}$ \\
\hline 884 & 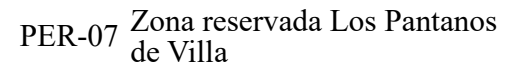 & Lima & 263 & $20 / 01 / 1997$ & $12^{\circ} 12^{\prime} \mathrm{S} 76^{\circ} 59^{\prime} \mathrm{O}$ \\
\hline 1174 & $\begin{array}{l}\text { PER-08 Complejo de humedales del } \\
\text { Abanico del río Pastaza }\end{array}$ & Loreto & 3827329 & $05 / 06 / 2002$ & $04^{\circ} 00^{\prime} \mathrm{S} 75^{\circ} 25^{\prime} \mathrm{O}$ \\
\hline 1317 & PER-09 $\begin{array}{l}\text { Bofedales y Laguna de } \\
\text { Salinas }\end{array}$ & Arequipa & 17657 & $28 / 10 / 2003$ & $16^{\circ} 22^{\prime} \mathrm{S} 71^{\circ} 08^{\prime} \mathrm{O}$ \\
\hline 1318 & $\begin{array}{l}\text { PER-10 Laguna del Indio - Dique de } \\
\text { los españoles }\end{array}$ & Arequipa & 502 & $28 / 10 / 2003$ & $15^{\circ} 46^{\prime} \mathrm{S} 71^{\circ} 03^{\prime} \mathrm{O}$ \\
\hline 1627 & PER-11 Humedal Lucre - Huacarpay & Cuzco & 1979 & $23 / 09 / 2006$ & $13^{\circ} 37^{\prime} \mathrm{S} 71^{\circ} 44^{\prime} \mathrm{O}$ \\
\hline 1691 & PER-12 Lagunas Las Arreviatadas & Cajamarca & 1250 & $15 / 07 / 2007$ & $05^{\circ} 14^{\prime} \mathrm{S} 79^{\circ} 17^{\prime} \mathrm{O}$ \\
\hline 1811 & $\begin{array}{l}\text { PER-013 Manglares de San Pedro de } \\
\text { Vice }\end{array}$ & Piura & 3399 & $12 / 06 / 2008$ & $05^{\circ} 31^{\prime} \mathrm{S} 80^{\circ} 53^{\prime} \mathrm{O}$ \\
\hline
\end{tabular}

Estas zonas al formar parte de los sitios Ramsar, cobran importancia por percibir fondos de cooperaciones internacionales e intercambio de experiencias, con el fin de promover la conservación y uso sostenible de los humedales ${ }^{1}$.

En ausencia de estudios que contengan un análisis exhaustivo sobre la Valoración Económica Ambiental de los Bofedales en Huancavelica, es que la presente investigación se convierte en una herramienta de información que podría ayudar a la toma de decisiones que beneficien las comunidades del ámbito de estudio y se coadyuve a una gestión sostenible de los bofedales.

El presente estudio fue diseñado con el objetivo de valorar los servicios ambientales de provisión de agua y almacenamiento de agua y carbono en el distrito de Pilpichaca, provincia de Huaytará departamento de Huancavelica, para determinar la importancia económica y ambiental.

\footnotetext{
${ }^{1}$ http://www.ramsar.org/es/acerca-de/laconvenci $\% \mathrm{C} 3 \% \mathrm{~B} 3 n$-de-ramsar-y-su-misi $\% \mathrm{C} 3 \% \mathrm{~B} 3 \mathrm{n}$
}

\section{Materiales y métodos}

Se consideró las 8369,67 ha de bofedales definidos en el Zonificación Económica Ecológica del Gobierno Regional de Huancavelica (2014). También, se consideró el número total de llamas y alpacas por comunidad involucrada de acuerdo al Gobierno Regional de Huancavelica para el año 2013.

Para la valorización económica de los bofedales, se utilizó el concepto de Valor Económico Total, el cual diferencia entre los valores de uso y de no uso, donde el primero significa una interacción del ser humano y el recurso; los segundos son valores actuales $\mathrm{y}$ potenciales relacionados con un recurso ambiental que existe continuamente y no tiene que ver con su utilización. Asimismo, los valores de uso se dividen en directos e indirectos donde la valoración del bofedal se realizará en su uso más eficiente, la cual define una valoración parcial del uso de los bofedales dada por la actividad ganadera, donde esta última compite con la conservación del bofedales. En la Figura 2, se presenta el flujo del análisis de valoración. 


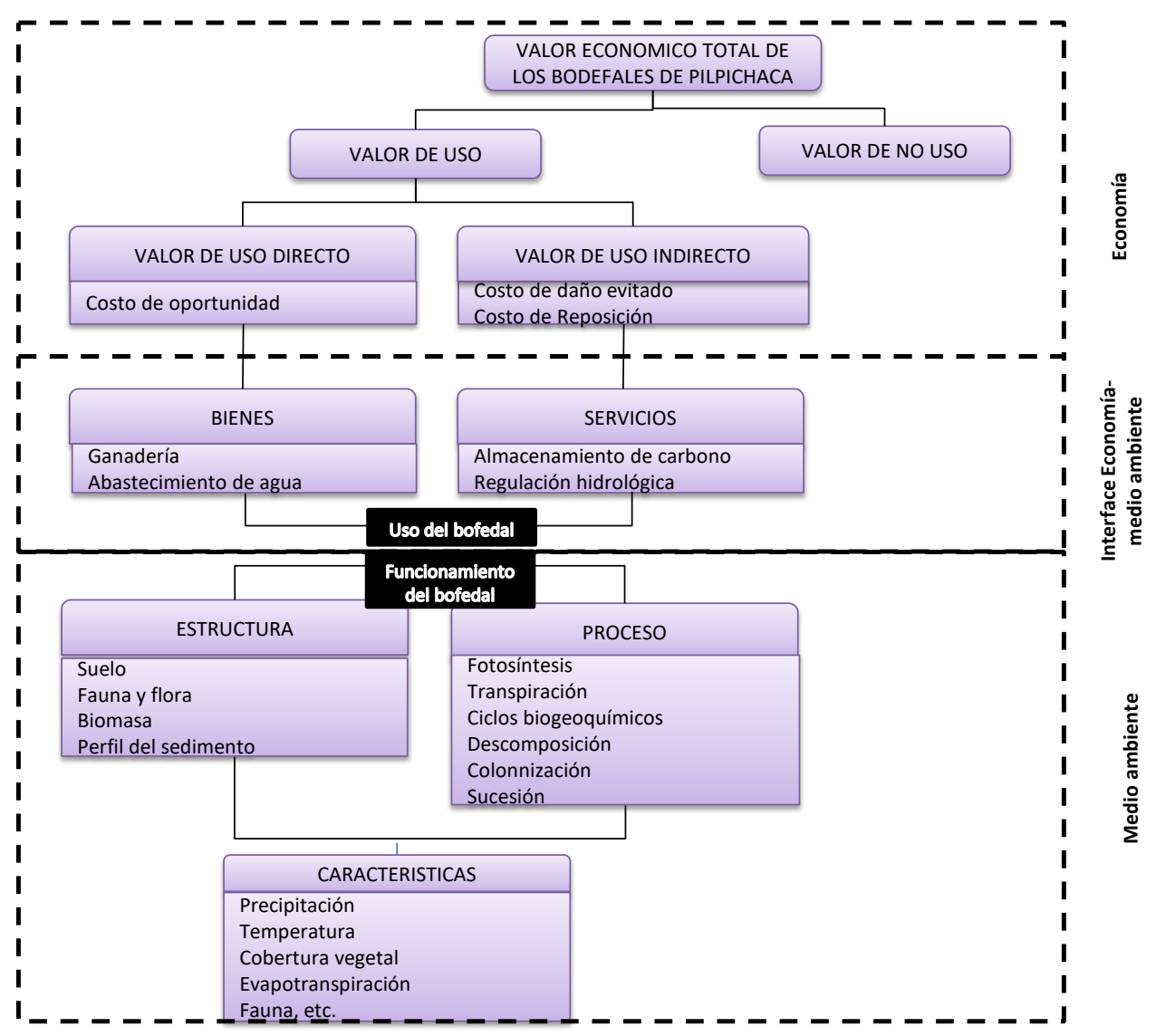

Figura 2: Estructura de valoración económica del estudio

Como parte del análisis se utilizaron diferentes técnicas y métodos económicos comúnmente utilizados (Perez, 2008), los cuales son: Método de la Productividad, Precio de mercado, Costo de oportunidad, Costo de daño evitado y Costo de reposición.

Esquematización de la valoración del servicio ambiental de provisión de agua

Para el análisis del servicio ambiental de provisión de agua se usó la siguiente fórmula (Barrantes y Vega, 2001):

$$
V P=\sum_{i=1}^{n} \frac{\alpha i \beta i A b i}{O d_{i}}
$$

Donde:
$V P=$ Valor de la productividad hídrica del bofedal (US $\left.\$ / \mathrm{m}^{3}\right)$.

$\beta \mathrm{i}=$ Costo de oportunidad de la ganadería (US\$/ha/año).

Abi = Area bajo cobertura de bofedal (ha).

Odi $=$ Oferta disponible captada por el bofedal ( $\left.\mathrm{m}^{3} / \mathrm{año}\right)$.

$\alpha \mathrm{i}=$ Índice de protección hidrológico.

Se requiere un análisis de oferta hídrica la cual está dado por la siguiente fórmula (Barrantes y Vega, 2001):

$$
O T=\sum_{I=1}^{n} 0.001 P i * A
$$


Donde:

$O T=$ Oferta total hídrica en el área de importancia ( $\left.\mathrm{m}^{3} / \mathrm{año}\right)$.

$\mathrm{Pi}=$ Precipitación en el bofedal i (mm/año)

$\mathrm{A}=$ área del bofedal $\mathrm{i}\left(\mathrm{m}^{2}\right)$.

Para efectos del análisis de la provisión de agua se requiere contar con el valor determinado de la oferta hídrica disponible el cual pasa por un análisis de evapotranspiración de Barrantes y Vega (2001):

$$
O d=\sum_{i=1}^{n}(O T) i-0.001 E T i * A i
$$

Donde:

Od = Oferta hídrica disponible en el área de importancia ( $\left.\mathrm{m}^{3} / \mathrm{año}\right)$.

ETi = Evapotranspiración en el área de importancia (mm/año).

Ai $=$ Area del bofedal i $\left(\mathrm{m}^{2}\right)$.
A continuación, se presentan las fórmulas determinadas a partir Holdridge (1978):

EVTp $=58,93 * \mathrm{~T}$

EVT $=$ Evapotranspiración

$\mathrm{RE}=E V T r / p$

$\mathrm{F} \quad=E V T r / E V T p$

Donde:

$\mathrm{T}=$ Temperatura

EVTp $=$ Evapotranspiración potencial

$\mathrm{P} \quad=$ Precipitación

EVTr $=$ Evapotranspiración real

$\mathrm{RE} \quad=$ Razón evapotranspiración potencial y precipitación

$\mathrm{F} \quad=$ Relación EVTr y EVTp

Fórmula de Holdridge (1978) en Barrantes y Vega (2001).

Se complementó el análisis del servicio ambiental de provisión de agua con la evaluación del costo de oportunidad de la actividad económica que compite con el uso del bofedal, dada por la siguiente expresión:

$$
C O=\frac{\sum_{i=1}^{j}\left\{[(W t)]_{r}-\sum_{i=1}^{j}\left\{[(W a)]_{r}-C p_{r}\right\}_{r}\right\}}{A}
$$

Donde:

$W t_{r}=$ Ingreso total por la venta del producto i

$C t_{r}=$ Costos efectivos de producción de i para la venta

Costo de oportunidad de $W a=$ Ingreso total por venta del subproducto $\mathrm{i}$ la ganadería

$C p_{r}=$ Costos de producción de subproductos

$\mathrm{A}=$ Superficie de pastoreo de la ganadería/ha (Barragan, 2008) 


\section{Almacenamiento de agua en el suelo de los bofedales}

El servicio ambiental de almacenamiento de agua en el suelo de los bofedales actúa como un gran reservorio natural que regula los flujos del ciclo hidrológico (Célleri, 2009; Buytaert et al., 2006; Biao et al., 2010), la cual puede compararse con la capacidad total de un reservorio o represa. Este valor puede ser calculado considerando los costos unitarios de construcción de una represa (Biao et al., 2010). El método utilizado es el del costo de reposición la cual considera un proyecto que puede sustituir el servicio ambiental (Pérez, 2008). La fórmula utilizada es la siguiente:

$$
\underset{\text { represa) }}{V_{\text {(almacenamientoagua) }}}=\partial * \mathrm{~A}(10000) * \rho *(0.01) * \mathrm{C}_{\text {(unit }}
$$

Dónde:

$V$ Es el valor en dólares (\$) del servicio ambiental hídrico de almacenamiento de agua.

$\partial$ Es el porcentaje de almacenamiento de agua en el suelo de los bofedales.

$A$ Es la superficie de bofedales medida en has dentro la zona de estudio.

$p$ Es el nivel freático del suelo medido en $\mathrm{cm}$ para la zona de bofedales.

$C$ Es el costo unitario en $\left(\$ / \mathrm{m}^{3}\right)$ para una represa.

La información referida al costo unitario de la represa proviene del estudio de factibilidad de la Presa Tambo perteneciente al Proyecto Tambo Ccaracocha.

\section{Almacenamiento de carbono en el suelo de los bofedales}

Para este análisis se utilizaron los datos que fueron proveídos por el Gobierno Regional de Huancavelica. El método utilizado es el costo del daño evitado a nivel global. Este costo consiste en el valor del beneficio social global que origina mitigar los Gases de Efecto Invernadero y evitar impactos asociados al cambio climático. Por otro lado, para hacer comparativo el valor de este servicio con los otros dos servicios ambientales de los bofedales se utiliza el método precios de mercado, donde al determinar el volumen de carbono en el suelo de bofedales será multiplicado por el precio de mercado al cual se cotiza. A continuación, se muestra la fórmula a utilizar:

Vcarbono $=$ Contenido $C$ en bofedales (ton $\left.\mathrm{CO}_{2} / \mathrm{ha}\right) *$ costo social del $\mathrm{C}\left(\$ /\right.$ ton $\left.\mathrm{CO}_{2}\right)$

Para determinar la primera parte de la ecuación se utiliza:

Contenido $C$ en bofedales (ton $\mathrm{CO}_{2} /$ ha $)=(\rho * h * C * 100) * 44 / 12 \quad$ Ecuación 15

Dónde:

$p$ Es la densidad del suelo medido en ( $\mathrm{g} /$ $\mathrm{cm}^{3}$ ).

$h$ Es la profundidad a la cual se tomó la muestra $(\mathrm{cm})$.

$C$ Es el porcentaje en peso de carbono orgánico en el suelo. La relación 44/12 proviene de la relación de estequiometria de pesos moleculares con la finalidad de conocer cuánto $\mathrm{CO}_{2}$ puede originarse por la descomposición del carbono orgánico. El factor 100 es el resultante de la compensación de las unidades: $\mathrm{cm}$, $\mathrm{m}$ y ha.

\section{Definición de escenarios}

En la zona de estudio, existe un conflicto por el agua entre los departamentos de Huancavelica e Ica, la misma que implica obras civiles de conducción del agua de las alturas hacia los valles de Ica, que afecta el curso natural del agua y por ende la existencia de los bofedales. En ese sentido, se plantea escenarios a fin de mostrar cual es la afectación de una intervención en uno varios parámetros respecto al escenario real. En la Tabla 4 se presentan los escenarios analizados. 
Tabla 4: Escenarios de producto del análisis del estudio

\begin{tabular}{|c|c|c|c|c|}
\hline \multirow[b]{2}{*}{$\begin{array}{l}\text { Valor del servicio } \\
\text { ambiental }\end{array}$} & \multirow[b]{2}{*}{ Escenario Real } & \multirow[b]{2}{*}{$\begin{array}{l}\text { Escenario } \\
\text { sostenible de carga } \\
\text { animal }\end{array}$} & \multicolumn{2}{|c|}{ Escenario de intervención } \\
\hline & & & $\begin{array}{l}\text { Reducción de } \\
\text { bofedal en } 20 \% \text {. }\end{array}$ & $\begin{array}{l}\text { Reducción } \\
\text { de bofedal y } \\
\text { afectación de } \\
\text { parámetros de } \\
\text { análisis. }\end{array}$ \\
\hline \multirow[b]{3}{*}{ Provisión de agua } & \multirow[b]{3}{*}{ Usando data real } & Solo llama & \multirow{3}{*}{$\begin{array}{l}\text { Se usará el } \\
\text { mismo número de } \\
\text { cabezas, mismos } \\
\text { ingresos y costos, } \\
\text { mismos valores } \\
\text { de precipitación y } \\
\text { evapotranspiración } \\
\text { en menor área de } \\
\text { bofedal. }\end{array}$} & \multirow{3}{*}{$\begin{array}{l}\text { Se considera } 20 \% \\
\text { menos de área } \\
\text { de bofedal y una } \\
\text { reducción del } 10 \% \\
\text { de la población } \\
\text { de alpacas y } \\
\text { llamas respecto al } \\
\text { escenario real. }\end{array}$} \\
\hline & & Solo alpaca & & \\
\hline & & $\begin{array}{l}\text { Carga recomendada } \\
\text { llama y alpaca }\end{array}$ & & \\
\hline $\begin{array}{l}\text { Almacenamiento } \\
\text { de agua }\end{array}$ & $\begin{array}{l}\text { Usando data real, } \\
\text { donde el parámetro } \\
\text { porcentaje de } \\
\text { almacenamiento de } \\
\text { agua en el bofedal es } \\
\text { igual a } 86,07 \% \text {. }\end{array}$ & & $\begin{array}{l}\text { Se considera todos } \\
\text { los parámetros } \\
\text { reales igual que el } \\
\text { escenario real pero } \\
\text { con un menor área } \\
\text { de bofedal. }\end{array}$ & $\begin{array}{l}\text { Se considera } \\
20 \% \text { menos de } \\
\text { área de fofedal } \\
\text { y una reducción } \\
\text { del parámetro } \\
\text { porcentaje de } \\
\text { almacenamiento de } \\
\text { agua en el suelo de } \\
\text { bofedales pasando } \\
\text { de un } 86,07 \% \text { a } \\
76,07 \% \text {. }\end{array}$ \\
\hline $\begin{array}{l}\text { Almacenamiento } \\
\text { de carbono }\end{array}$ & $\begin{array}{l}\text { Usando data real, } \\
\text { donde el parámetro } \\
\% \text { de carbono } \\
\text { orgánico en el suelo } \\
\text { es igual a } 24,89 \% \text {. }\end{array}$ & & $\begin{array}{l}\text { Se considera todos } \\
\text { los parámetros } \\
\text { reales igual que el } \\
\text { escenario real pero } \\
\text { con un menor área } \\
\text { de bofedal. }\end{array}$ & $\begin{array}{ll}\text { Se considera } & 20 \% \\
\text { menos de área } & \\
\text { de fofedal y una } \\
\text { reducción } \\
\text { parámetro del } \\
\text { carbono orgánico } \\
\text { en el suelo pasando } \\
\text { de un 24,89\% a } \\
14,89 \% \text {. }\end{array}$ \\
\hline
\end{tabular}

Fuente: Elaboración propia, escenarios de intervención son supuestos para mostrar los cambios en caso se diera cualquier de los cambios

\section{Resultados y discusión}

\section{Escenario real}

La cantidad total de alpacas y llamas presentes en la zona de estudio asciende a 71137 y 6231 unidad animal generando un ingreso bruto de US\$ 8634560 y US\$ 456 582 respectivamente por la venta del animal, US\$ 23146 por fibra de alpaca y US\$ 1073 por fibra de llamas, US\$10 155 por la carne de alpaca y US\$ 580 por la carne de llama, US\$ 1132 por el cuero de alpaca y US\$ 22 por el cuero de llama, todos estos productos con destino al mercado. Asimismo, US\$ 7715 por fibra de alpaca y US\$ 189 por fibra de llamas, US\$ 6770 por la carne de alpaca y US\$ 870 por la carne de llama, US\$ 1698 por el cuero de alpaca y US\$ 51 por el cuero de llama, todos estos productos para autoconsumo. Se descuenta al ingreso bruto total el costo por crianza de las especies pecuarias, dando como resultado un ingreso neto total de US\$3 492856 , donde el beneficio económico proviene de la comercialización del animal vivo, las fibras, la carne y el cuero como subproductos.

El costo-beneficio por hectárea es alrededor de US\$417,32 producto de la división de US\$3 492856 entre 8 369,67 ha de bofedal, el cual representa el Valor Económico Parcial por unidad de área que los pobladores obtienen por dedicarse a la comercialización de alpacas, llamas y sus respectivos subproductos. 
Del balance hídrico (oferta hídrica disponible) se obtuvo que el valor de productividad hídrica es US $\$ 0,05 \mathrm{~m}^{3}$ de agua, que expresado como un valor total para los bofedales de la zona de estudio es equivalente a US\$ 2759 355,92. Asimismo, en los bofedales, las especies ganaderas compiten con el uso natural del suelo en tanto el pisoteo y los hábitos alimenticios de las alpacas y llamas perjudican la cobertura vegetal y disminuyen la productividad hídrica. De igual manera la cobertura total se expresa en términos monetarios de US\$ 733 499,68. En la Figura 3 se presenta la relación del Valor de Productividad (VP) y Valor de Cobertura (VC).

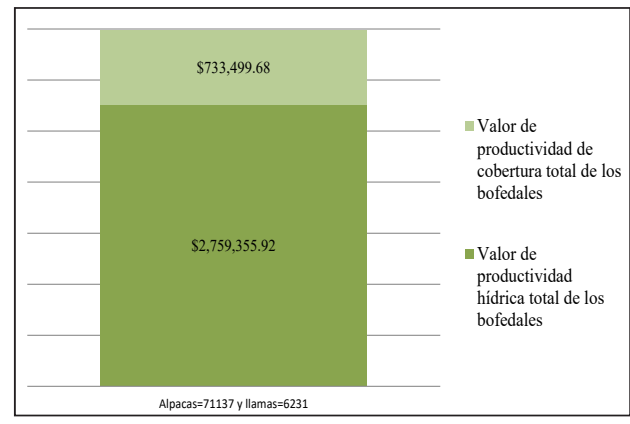

Figura 3: Relación del VP y VC para el área de bofedales - Escenario real

El VP excede es 3,8 veces más VC lo cual indica que aparentemente es más rentable el establecimiento de un valor económico en función a la productividad hídrica. Sin embargo, disgregar ambos servicios ecosistémicos es imposible ya que están vinculados por procesos ecológicos naturales.

\section{Escenario de intervención: reducción del área de bofedal}

Se considera que el área de bofedal se reduce en un $20 \%$ producto de la intervención de manera sostenible, donde en términos de valor no habría variación respecto al escenario real dado, que al haberse reducido el área del bofedal también se redujo en la misma proporción la oferta hídrica y el valor de los otros servicios ambientales, sin afectar drásticamente los parámetros ambientales.

\section{Escenario de intervención: Reducción del área de bofedal y número de cabezas de ganado}

Se considera que el área de bofedal se redujo en un $20 \%$ producto de la intervención de las obras físicas de trasvase o conducción del agua de las zonas altas hacia las zonas bajas, modificando físicamente el área de los bofedales; afectando a los pobladores y por ende migran buscando otras oportunidades, abandonan la actividad ganadera. La cantidad total de alpacas y llamas presentes en la zona de estudio fue 64023 y 5608 unidad animal respectivamente. Del balance hídrico, el valor de productividad hídrica fue igual a US\$ $0,056 \mathrm{~m}^{3}$ de agua y como valor total para los bofedales fue igual a US\$ 2 $483420,33$.

Asimismo, las especies ganaderas compiten por el uso de suelo natural en el bofedal, en tanto el pisoteo y los hábitos alimenticios de las alpacas y llamas perjudican la cobertura vegetal y disminuyen la productividad hídrica. El valor de cobertura total es igual a US\$ 660149,71 . Es así, que huvo una variación en la misma proporción que se redujo el número de cabezas de ganado, más no por la reducción del área de bofedal al igual que los resultados del valor de los otros servicios ambientales. En la Figura 4, se muestra los escenarios analizados; siendo el valor de provisión de agua el que tiene mayor participación respecto al valor de almacenamiento de agua $\mathrm{y}$ almacenamiento de carbono.

\section{Comparación del valor del servicio ambiental de provisión de agua}

A un tipo de cambio igual a 2,9 soles por dólar se homogenizó todas las unidades para poder realizar la comparación. Un estudio en Ferrobamba muestra que el valor de la productividad hídrica del bofedal es mayor en comparación con las otras zonas, esto es debido a que el costo de oportunidad de la ganadería es mayor en Ferrobamba comparado con Pilpichaca, Oña-Nabón y Tungurahua. Cabe resaltar que Ferrobamba y Pilpichaca son considerados como parte del Corredor Económico de los Camélidos Sudamericanos (Tabla 5). 

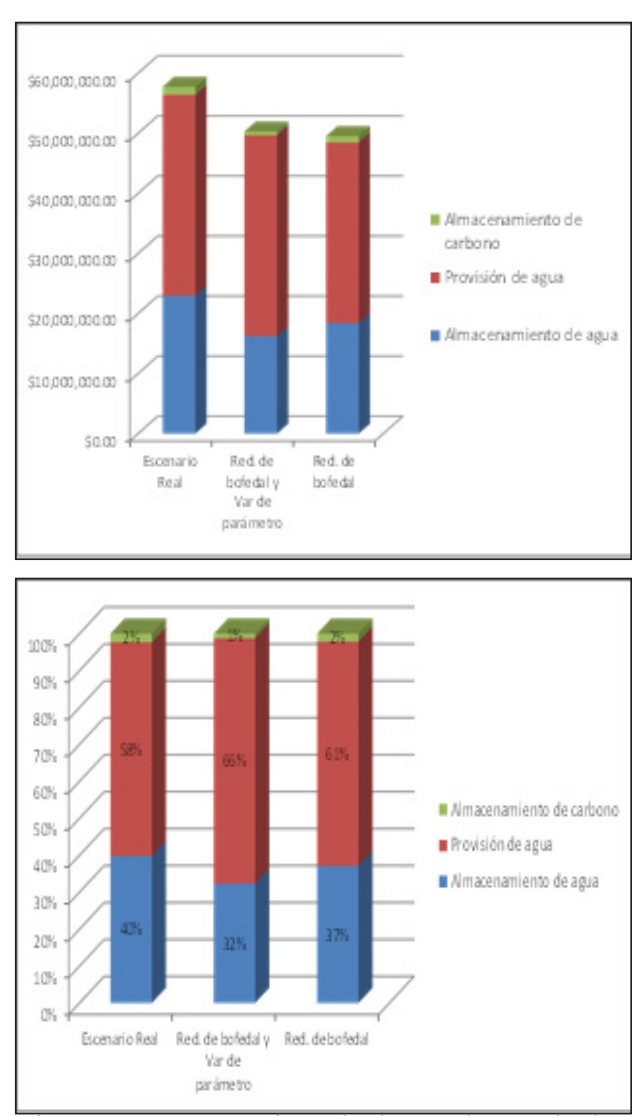

Figura 4: Integración de los valores de los servicios ambientales de provisión de agua, almacenamiento de agua y almacenamiento de carbono
Comparación del valor del servicio ambiental de almacenamiento de agua

El valor unitario del almacenamiento de agua en Pilpichaca es menor en un $15 \%$ aproximadamente en comparación con los resultados obtenidos en Oña-Nabón y Tungurahua, esto debido a que el costo de represamiento es menor en Pilpichaca comparado con las otras zonas (Tabla 6).

\section{Comparación del valor del servicio} ambiental de almacenamiento de carbono

El valor unitario obtenido en Pilpichaca es igual a $160,83 \$ /$ ha frente a $13340,62 \$ /$ ha y 7787,26 \$/ha de Oña - Nabón y Tungurahua respectivamente. Esta diferencia es por el valor del costo social del carbono considerado. Es decir, en el año 2014 el costo social fue del carbono fue mayor respecto al año 2011. Esta diferencia se debe a muchos factores, pues a lo largo del tiempo se viene desarrollando y fortaleciendo el mercado del carbono y por ello la fluctuación (Tabla 7).

En resumen, los bofedales son vitales para el servicio ambiental hídrico, no solo por el volumen de agua que almacenan sino por la función de regulación hídrica que tienen, dado que existe una alta precipitación $(942,89 \mathrm{~mm})$ y una baja evapotranspiración lo cual nos lleva a una saturación permanente de los suelos de los bofedales, bajo esta situación se dan altos rendimientos hídricos que se traducen en provisión de agua. La función de regulación de los bofedales permite que la variabilidad de caudales en épocas de avenidas y estiajes no se conviertan en excesos o déficit de agua, sino que permite almacenar agua y proveerla en la época seca (Stolk et al., 2006).

Tabla 5: Comparación del valor del servicio ambiental provisión de agua con otros resultados

\begin{tabular}{lcccc}
\hline Autores & \multicolumn{2}{c}{ Castro (2011) } & $\begin{array}{c}\text { Arenas y Pineda } \\
(2011)\end{array}$ & $\begin{array}{c}\text { Crispin } \\
(2015)\end{array}$ \\
\hline Rubro comparado & Oña-Nabón & Tungurahua & Ferrobamba & Pilpichaca \\
Valor de la productividad hídrica del bofedal & 0,03 & 0,02 & 0,15 & 0,05 \\
$\left(\$ / \mathrm{m}^{3}\right)$ & 505,1 & 800 & 862,2 & 942,89 \\
Precipitación (mm/año) & 218 & 1971 & 469 & 8369,67 \\
Area de bofedal (ha) & & & &
\end{tabular}

Fuente: Elaboración en base a la revisión de literatura 
Tabla 6: Comparación del valor del servicio ambiental almacenamiento de agua en los suelos de los bofedales

\begin{tabular}{lccc}
\hline Autores & \multicolumn{2}{c}{ Castro $(2011)$} & Crispin $(2015)$ \\
\hline Rubro comparado & Oña-Nabón & Tungurahua & Pilpichaca \\
Valor unitario del almacenamiento de agua $(\$ / \mathrm{ha})$ & 3196,9 & 3299,21 & 2744,39 \\
Costo unitario de represamiento del agua $\left(\$ / \mathrm{m}^{3}\right)$ & 1,86 & 1,86 & 1,59 \\
\hline
\end{tabular}

Fuente: Elaboración Propia en base a la revisión de literatura

Tabla 7: Comparación del valor del servicio ambiental almacenamiento de carbono en los suelos de los bofedales

\begin{tabular}{lccc}
\hline Autores & \multicolumn{2}{c}{ Castro $(2011)$} & Crispin $(2015)$ \\
\hline Rubro comparado & Oña-Nabón & Tungurahua & Pilpichaca \\
Valor unitario del almacenamiento de carbono $(\$ /$ & 13340,62 & 7787,26 & 160,83 \\
ha) & 15 & 15 & 0,31 \\
Costo social del carbono $\left(\$ / \mathrm{t} \mathrm{CO}_{2}\right)$ &
\end{tabular}

Fuente: Elaboración en base a la revisión de literatura

Por otro lado, la alta carga de materia orgánica en el suelo de los bofedales, da un servicio ambiental de almacenamiento de carbono, ya que al almacenarse se evita la descomposición y por ende la emisión de $\mathrm{CO}_{2}$ el cual es considerado un Gas de Efecto Invernadero (GEI), donde el servicio ambiental de almacenamiento de carbono es un valor de uso indirecto dado que da pauta a una regulación de gases y su influencia en el cambio climático. De lo mencionado, se concluye que la importancia de los bofedales radica en la provisión y almacenamiento de agua y en menor medida la de almacenamiento de carbono el cual es totalmente diferente a lo obtenido por Castro (2011).

Del análisis comparativo, si solo se toma en cuenta los valores analizados por Castro (2011), el valor del servicio de almacenamiento de carbono es mayor comparado con los otros servicios; sin embargo, en el presente estudio el valor del servicio de almacenamiento de carbono menor en comparación con los otros servicios. Pero independientemente del resultado específico de la valoración por servicio ambiental, lo cierto es que existe un valor de los servicios ambientales de los bofedales, ya sea dado por el costo de oportunidad de la ganadería la cual se materializa en el servicio de provisión de agua el cual puede ser mayor comparado con los otros servicios; o donde Servicio de Almacenamiento de Carbono es mayor comparado con los otros servicios. Esto es debido a las fluctuaciones del precio del carbono y de ahí la importancia de la fijación de carbono en el suelo de los bofedales. Visto de cualquier ángulo, sea cual fuese el servicio ambiental de mayor valor, lo cierto es que hay una gran importancia de la existencia de los bofedales por los servicios ambientales que provee.

A partir de estos resultados se apela a formalizar que los bofedales sean parte integrante de los Sitios Ramsar y con ello una mayor oportunidad de promoción sobre la conservación y uso sostenible de los bofedales de Pilpichaca.

\section{Porqué los bofedales de Pilpichaca deben ser incluidos como Sitio Ramsar en el Perú}

- De los 13 sitios Ramsar que tiene el Perú, ninguno está ubicado en la zona altoandina por encima de los 3000m.s.n.m.

- Permitiría la canalización de fondos de la cooperación internacional e intercambio de experiencias que permita la conservación y el uso racional de todos los humedales. Ademas de contribuir con el desarrollo sostenible.

- Los gobiernos locales podrían servirse de una herramienta política para poder orientar los esfuerzos para la protección 
y uso racional de los bofedales, de tal manera que no afecte los ingresos de las familias Altoandinas.

- Los gobiernos locales tendrían una base legal construir un sistema de pagos ambientales que involucre al Gobierno Regional de Ica y Huancavelica. Además, ser una alternativa de solución al conflicto por el agua, el cual es un tema conocido a nivel de ambas regiones

\section{Conclusiones}

- Se ha evaluado y determinado que existe una importancia económica y ambiental de los bofedales de Pilpichaca el cual está dado por el valor de provisión de agua, el valor de almacenamiento de agua y el valor de almacenamiento de carbono.

- El valor de provisión de agua como participación del valor total es siempre mayor en cualquiera de los escenarios analizados. El valor de almacenamiento de agua para el escenario real asciende a US\$ 22969 667, para el escenario de intervención con variación de área de bofedal y parámetros ambientales es igual a US\$ 16240758 y para el escenario donde varia el área de bofedal es igual a US\$ 18375733 , donde se puede concluir que el efecto de una variación de área de bofedal y los parámetros ambientales es mayor en comparación si solo varía el área de bofedal. Asimismo, este valor es el segundo de mayor importancia después del de provisión de agua.

- En relación al valor de almacenamiento de carbono el escenario real asciende a US\$ 1346 068; mientras que para un escenario de intervención con reducción de área de bofedal y parámetros ambientales es igual a US\$ 626824 y para el escenario de solo una variación en área de bofedal el valor asciende a US\$ 1076 854. Es el de menor valor económico, esto debido a que el precio de carbono utilizado para el presente análisis es bastante menor, esto obedece a que los derechos de emisión (CER) es fluctuante en el tiempo y depende de muchos factores.

- Se Justifica declarar un sitio Ramsar a los bofedales de Pilpichaca dada la importancia económica, ambiental, social y política. Ambiental por la función de regulación hídrica, almacenamiento de agua y carbono; económico por que existe una actividad económica ganadera vinculado con los bofedales, la misma que a la fecha falta mucho por mejorar para ser una actividad económica sostenible; social, porque se podría orientar esfuerzos tanto nacional como internacional para proteger los bofedales y promover el uso racional de los mismos la cual involucre a las comunidades y por último política, dado que al contar con la declaración de un Sitio Ramsar los bofedales de Huancavelica se pueden justificar varias herramientas de intervención y negociación con otras regiones aledañas con el afán de establecer un sistema de pagos por servicios ambientales como por ejemplo Huancavelica e Ica.

\section{Literatura citada}

Arenas, F; Pinedo, P. 2013. Valoración Económica Ambiental de los Bofedales de la Subcuenca del Río Ferrobamba Apurímac. Tesis Maestría, Universidad Nacional Agraria La Molina, Lima. Perú. 167 pp.

Barrantes, G; Vega, M. 2001. Evaluación del Servicio Ambiental Hídrico en la Cuenca del Río Savegre con fines de Ordenamiento Territorial. Desarrollo Sostenible de la Cuenca hidrográfica del Río Savegre. Costa Rica.

Biao, Z; Wenhua, L; Gaodi, x; Yu, X. 2010. Water conservation of forest ecosystems in Beijing and its value. Ecological Economics 69: 1416-1426.

Buytaert, W; Célleri, R; De Bièvre, B; Hofstede, R; Cisneros, F; Wyseure, G; Deckers, J. 2006. Human impact on the hydrology of the Andean páramos. Earth Science Reviews 79: 53-72.

Castro, M. 2011. Una valoración Económica del almacenamiento de agua y carbono en los bofedales de los Páramos Ecuatorianos.

Célleri, R. 2009. Estado del conocimiento técnico sobre los servicios ambientales 
hidrológicos generados en los Andes. Servicios ambientales para la conservación de los recursos hídricos: lecciones desde los Andes. Síntesis Regional CONDESAN.

Crispin, M. 2015. Valoración económica ambiental de los bofedales del distrito de Pilpichaca Huancavelica Perú. Tesis de Magister Scientiae, Universidad Nacional Agraria La molina, Lima. Perú. $167 \mathrm{pp}$.

Custred;Glynn. 1997. Las punas de los Andes Centrales. en: Flores Ochoa. Jorge, Comp. Pastores de puna. Uywamichiqpunarunakuna. LIMA: IEP; 1977: 55-86.

Gobierno Regional de Huancavelica. 2014. Zonificación Ecológica y Económica de Huancavelica.

Holdridge, LR. 1978. El diagrama de las zonas de vida. En ecología basada en zonas de vida. San José, IICA. p. 13-28.

Gil, JE. 2011. Bofedal: Humedal altoandino de importancia para el desarrollo de la Región Cusco. Cusco.

Pérez, O. 2008. Valoración económica de los Recursos Naturales y del Ambiente - Importancia y limitaciones, metodología y técnicas, estudios de caso $\mathrm{y}$ aplicaciones.

PNUMA (Programa de las Naciones Unidas para el Medio Ambiente Oficina Regional para América Latina y el Caribe). 2005. Borrador 6.1. Evaluación de los Ecosistemas del Milenio. Informe técnico. $17 \mathrm{p}$.

Secretaría de la Convención de Ramsar. 2013. Manual de la Convención de Ramsar, 6ta edición. Ramsar. Irán.

Stolk, M.E.P.; Veweij, M.; Baker, C.; Oosterberg, W. 2006. Valoración Socioeconómica de los Humedales en América Latina y el Caribe. Wetlans International. Los países Bajos. Disponble en: http://www. estadis.net/3/actas/POS/04.\%20 E1\%20an\%C3\%A11isis\%20de\%20 Correspondencias $\% 20 y \% 201 \mathrm{a} \% 20$ valoraci $\%$ C $3 \%$ B 3 n $\% 20$ social $\% 20$ de $\% 201$ a $\% 20$ flor a $\% 20$ de $1 \% 20$ humedal $\% 20 \mathrm{el} \% 20$ Coroncoro $\% 20 \mathrm{de} \%$ 20Villavicencio.pdf 